\title{
Die deutsch-tschechischen Beziehungen und die Präsidentschaftswahl 2013
}

\author{
Lukáš Novotný1,* \\ 1 Politology and Philosophy Department, Philosophical Faculty, J. E. Purkyne University in Usti n. Labem, Czech Republic \\ * E-Mail: novotny.l@centrum.cz
}

\section{Zusammenfassung}

Ziel dieser Studie ist es, die Rolle der deutsch-tschechischen Beziehungen bei der Präsidentschaftswahl 20I3 in Tschechien zu analysieren. Insbesondere geht es um die Frage, inwieweit Geschichtsthemen wie die Vertreibung der deutschen Bevölkerung sowie die Benes-Dekrete eine Rolle im Wahlkampf spielten. Es zeigt sich, dass sie das Ergebnis der Wahl nicht in entscheidendem Ausmaß beeinflusst haben, was nicht zuletzt an dem geringen Abstand zwischen den beiden verbleibenden Kandidaten in der zweiten Runde ersichtlich wurde. Dies ist bemerkenswert auch deshalb, wenn man sich vor Augen hält, welch explosive Rolle Vertreibung und Dekrete vor einigen Jahren in Tschechien noch hatten.

\section{Schlüsselwörter:}

deutsch-tschechische Beziehungen, Präsidentschaftswahl, Beneš-Dekrete

\section{Czech-German relations and presidential elections 2013}

\begin{abstract}
This study examines the role of Czech-German relations in the debates during the 2013 presidential election campaign in the Czech Republic. While issues especially like the displacement of German-speaking population and the validity or the invalidity of the Benes Decrees still have some kind of social relevance, their actual influence on the election result was negligible. This can be seen also from the relatively close score of the two remaining candidates of the second round of the campaign. Given the explosiveness of the topic just a few years ago, this points to the fact that the relationship between the two nations are normalizing.
\end{abstract}

\section{Keywords:}

Czech-German relations, presidential election, Beneš Decrees

The author has declared that no competing interests exist. 


\section{Einleitung ${ }^{1}$}

Das Thema deutsch-tschechische Beziehungen taucht in der tschechischen Politik immer wieder in regelmäßigen Abständen seit Anfang der 9oer Jahre auf, als in der Öffentlichkeit die Diskussion über strittige Punkte der gemeinsamen Geschichte begann und als Deutschland und Tschechien sich infolge der Beendigung des Kalten Krieges auch genötigt sahen, ihre Beziehungen zueinander $\mathrm{zu}$ normalisieren. Der Ausgleich dieser vom Zweiten Weltkrieg und seinen Folgen beeinträchtigten gegenseitigen Beziehungen lag nicht nur in ihrem eigenen Interesse als Nachbarvölker in Mitteleuropa, sondern auch im Interesse weiterer Staaten. So wissen wir z. B., dass der Wortlaut der Deutsch-Tschechischen Erklärung über die gegenseitigen Beziehungen und deren künftige Entwicklung von der amerikanischen Diplomatie wesentlich beeinflusst wurde, die - nach den Worten des damaligen Staatssekretärs im US-Außenministerium Richard Holbrooke - interessiert daran war, „dass wir unsere Streitigkeiten beilegen“, worauf übrigens auch einer der früheren Unterhändler, der spätere tschechische Verteidigungsminister Alexandr Vondra hinweist (Vondra 2007, II).

Mit dieser Formulierung gemeint waren die Streitigkeiten im Zusammenhang mit der unterschiedlichen Interpretation der Kriegsereignisse, aber auch der Vor- und Nachkriegsereignisse (Houžvička 2015). Die Notwendigkeit, die deutsch-tschechischen Beziehungen zu normalisieren, war Mitte der 9oer Jahre beiden beteiligten Seiten (vor allem deren Außenministern und Staatspräsidenten) deutlich geworden, $u$. a. im Zusammenhang mit dem erwarteten EU-Beitritt der Tschechischen Republik, da die Beitrittsgespräche nicht durch Streitigkeiten und Emotionen auf beiden Seiten gestört werden sollten (Novotný 2015).

Dem Thema deutsch-tschechische Beziehungen begegnen wir hin und wieder in den öffentlichen Diskussionen beider Nationen. Allerdings sind es vor allem tschechische Politiker, die dieses Thema (konkret die Frage der Vertreibung der Sudetendeutschen und der sog. Präsidialdekrete) gern für ihre Zwecke aufgreifen, wenn sich damit ein politischer Punktegewinn erzielen lässt. Auf deutscher Seite findet sich eine ähnliche Instrumentalisierung vorwiegend in der Strategie mancher Vereine, jedoch nicht bei politischen Parteien oder Vertretern des Staates, und keinesfalls spielen diese Themen eine Rolle in Kampagnen und Wahlkämpfen. Anders ist das in der Politszene Tschechiens, obwohl es auch hier Beispiele für Situationen gibt, in denen die deutsch-tschechischen Beziehungen nicht zum Thema in einer Kampagne wurden. ${ }^{2}$ Im Verlauf des Jahres 2013

I Diese Untersuchung wurde im Rahmen des Projekts „EU-Politik Deutschlands" dank der großzügigen Förderung durch das Unternehmen Severočeské doly sowie die Jan Evangelista Purkyně Universität in Ústí nad Labem und die Technische Universität Chemnitz ermöglicht.

2 Zum Beispiel vor den Wahlen im Jahr 1996, in der Zeit als die Deutsch- konnte man allerdings zwei gegensätzliche Fälle der Thematisierung der deutsch-tschechischen Beziehungen verzeichnen: einerseits die versöhnliche Rede des damaligen Premierministers Petr Nečas im Bayerischen Landtag darüber, wie notwendig es sei, die gemeinsame Vergangenheit zu reflektieren, Lehren aus ihr zu ziehen und „für eine gemeinsame Zukunft zu arbeiten"3, andererseits die Kampagne vor der zweiten Runde der Präsidentschaftswahl, in der die historischen Themen Beneš-Dekrete und Vertreibung der Sudetendeutschen stark anklangen, ähnlich wie in der hektischen Atmosphäre der 9oer Jahre des 20. Jh. (Novotný/Houžvička 2007; Perzi 2003; CoudenhoveKalergi/Rathkolb 20I3).

Der vorliegende Artikel befasst sich konkret mit der Rolle deutsch-tschechischer Themen während des Wahlkampfes vor der Präsidentschaftswahl. Ausgegangen wird dabei von der zweiten Wahlrunde, da in der ersten diese Fragen praktisch nicht zur Sprache kamen. Wesentlich jedoch ist, dass vor allem die Fernsehdebatten vor dem zweiten Wahlgang die unbestritten größte Relevanz für den potenziellen Wähler hatten, und zwar dank ihres starken audiovisuellen Eindrucks. Erst die Polarisierung des Wahlkampfs in der zweiten Wahlrunde auf die Person des klaren Gewinners der ersten Runde, Miloš Zeman, und die Person seines Herausforderers, des entgegen den Ergebnissen der Meinungsumfragen zweitplatzierten Karl Schwarzenberg, ermöglichte, dass diese Fragen Wahlkampfthema wurden. Heute lässt sich nur konstruieren, „was wäre wenn“, aber das Wiederaufleben der Diskussion um die umstrittenen Punkte der deutsch-tschechischen Geschichte war sicherlich bedingt durch den Erfolg Karl Schwarzenbergs und dessen Aufstieg in die zweite Wahlrunde, ein Gegner, mit dem Miloš Zeman vielleicht nicht gerechnet hatte. Von diesem Moment an brauchten sich beide Wahlkampfteams nur noch auf den Gegenkandidaten und seine eventuellen Schwachstellen zu konzentrieren. Die Realität der Ergebnisse hatte M. Zeman vor vollendete Tatsachen gestellt und die deutsch-tschechischen Themen, einschließlich der deutschen bzw. österreichischen Geschichte der Familie Schwarzenberg, boten sich für den politischen Kampf um den Einzug in die Prager Burg direkt an.

Tschechische Erklärung ausgehandelt wurde, vermieden es die beiden stärksten Parteien, ODS und ČSSD, dieses brisante Thema zu verwenden (auch infolge des Einflusses der deutschen Schwesterparteien). Ausdrücklicher thematisiert wurden die deutsch-tschechischen Beziehungen von der ODS in den Wahlen 2002, aber auch da ging es nicht um Mobilisierung, sondern vielmehr um die Betonung „nationaler Interessen“ und de facto um die Ablehnung des deutschen Politik-Modells.

3 Der vollständige Wortlaut der Rede von Premier Nečas vor dem Bayerischen Landtag in München am 22. Februar 2013 hier: https://www.euroskop.cz/46/220I4/clanek/projev-petra-necasev-bavorskem-zemskem-snemu/ (Zugriff I2.I2. 20I5) 
Theoretischer Ausgangspunkt für den Aufsatz sind einerseits die Prinzipien des kollektiven Geschichtsgedächtnisses, wie sie Maurice Halbwachs formuliert hat und wie sie auch von weiteren Gedächtnissoziologen interpretiert werden. Es sind vor allem die selektive Beschaffenheit und das Auswählen von Aspekten der erlebten, aber auch tradierten Geschichte, die gemeinsam das kollektive Geschichtsgedächtnis bilden. Diese lassen sich auf die Realität des Wahlkampfs in den Präsidentschaftswahlen in der Tschechischen Republik für das Jahr 2013 zwar nur in einigen Aspekten anwenden, dennoch sind Halbwachs' Erkenntnisse instruktiv für die anschließende Interpretation dieser Themen. Andererseits wird diesem Aufsatz das Konzept der Europäisierung zu Grunde gelegt.

Häufig wird davon gesprochen, dass sich nicht nur die tschechische Politik, sondern auch die deutschtschechischen Beziehungen in der Phase der Europäisierung befinden. Es ist schwierig, diese Beziehungen genau zu definieren und ihre Wirksamkeit lässt sich kaum messen, für die Erfordernisse der vorliegenden Arbeit sollen sie als spezifische Auffassung verstanden werden, die die Existenz der Europäischen Union und die EU-Mitgliedschaft beider Länder berücksichtigt. Diese Situation stellt die gegenseitigen Beziehungen in den Kontext europäischer Geschichte und zeigt diese nicht in ihrem „Rohzustand" und ihrer Faszination nur für sich selbst, sondern verweist auf den versöhnlichen Geist der EU und auf die Notwendigkeit, solchen auch in das deutsch-tschechische Verhältnis hineinzutragen.

Die zentrale These der Arbeit ist somit, dass bei aller Aggressivität, mit der deutsch-tschechische Geschichtsthemen, und bedingt durch die Person Karl Schwarzenbergs auch österreichisch-tschechische Geschichtsthemen für den Wahlkampf genutzt wurden, die Europäisierung der öffentlichen Meinung sich als aktuell erwiesen hat. In der öffentlichen Meinung gelten nämlich diese historischen Fragen nicht weiter als umstritten. Wie sonst ließe sich das 45-Prozent-Ergebnis für Karl Schwarzenberg erklären? Bei einem hohen Maß an Sensibilität für deutsch-tschechische, vor allem mit der Vertreibung der Deutschen nach dem Zweiten Weltkrieg zusammenhängende Geschichtsthemen wäre das Ergebnis wohl deutlich ungünstiger ausgefallen. Denn der Wähler vernahm aus dem Munde des damaligen tschechischen Außenministers Schwarzenberg u. a. die Negierung der Gültigkeit der Präsidialdekrete und die Frage nach der Verhältnismäßigkeit der Vertreibung der Deutschen aus der Tschechoslowakei. Solche Äußerungen hätten in den 9oer-Jahren des vorigen Jahrhunderts mit Sicherheit zu einem starken Rückgang der Wählergunst geführt. Die Ergebnisse der zweiten Wahlrunde lassen jedoch den Schluss zu, dass einerseits die Ausfälle Zemans betreffend die deutsch-tschechische Geschichte, um Schwarzenbergs Position zu schwächen, und ei- nige unüberlegte Reaktionen Schwarzenbergs andererseits nicht die erwartete Wirkung auf den Wähler hatten.

Methodologischer Ausgangspunkt ist die Inhaltsanalyse dreier Fernsehdebatten zwischen den beiden Präsidentschaftskandidaten, die unmittelbar vor der Wahl stattfanden. Zwei Debatten wurden vom öffentlich-rechtlichen Tschechischen Fernsehen, eine Debatte wurde vom privaten Fernsehkanal Prima ausgestrahlt. Das Augenmerk richtet sich hierbei vor allem auf ausgewählte Kernpassagen zur Problematik der deutschtschechischen Beziehungen und auf die allgemeine Dynamik, die bei Themen aus diesem Themenbereich zu beobachten ist.

Technische Anmerkung zum Text: Wiederholt werden Aussagen der Präsidentschaftskandidaten bzw. der Moderatoren aus den einzelnen Fernsehdebatten zitiert, an diesem Stellen verzichtet der Autor auf Querverweise zum Internetportal des Tschechischen Fernsehens oder zum Server YouTube.com, wo diese Debatten in vollem Wortlaut wiedergegeben werden. ${ }^{4}$

\section{Theoretische Ansatzpunkte}

In letzter Zeit hat, besonders im Zusammenhang mit Oral History und deren Bedeutung in der Geschichtswissenschaft und in der Sozialwissenschaft allgemein, die Gedächtnissoziologie an Popularität gewonnen. Zu den führenden Denkern, die sich mit diesen Fragen beschäftigt haben, gehört der Franzose Maurice Halbwachs (1877-1945). Der Philosoph und in erster Linie Soziologe ist der Autor des Konzepts des kollektiven Gedächtnisses (La mémoire collective), an dem er in den $20 e r$ bis $40 e r$ Jahren des 20. Jh. arbeitete. Seine Theorie wurde sehr beliebt und deren theoretische Prinzipien zählen gerade im Zusammenhang mit der Gedächtnissoziologie zu den am häufigsten angewendeten (Halbwachs 1992; Egger 2003; Goff 1992). Bei ihrer Formulierung ging Halbwachs von soziologischen und philosophischen Ansätzen aus, die aufgrund seiner fachlichen Orientierung für ihn naheliegend waren. Halbwachs betont die soziale Bedingtheit des Gedächtnisses, damit grenzt er sich gegen Theorien ab, die das Kollektivgedächtnis als vererbbar definierten (Kreisslová 20I3). Halbwachs sieht die Kontinuität des kollektiv geteilten Bewusstseins nicht in der Biologie, sondern in der Kultur bedingt. Die spezielle Verhaltensweise, die der Mensch durch seine Zugehörigkeit zu einer bestimmten Gesellschaft und ihrer Kultur annimmt, ist seiner Ansicht nach nicht das Ergebnis der phylogenetischen Evolution. Halbwachs versteht das Gedächtnis vor allem als bestimmten inneren Raum, der mit jedem Einzelwesen im Prozess seiner Sozialisierung

4 Siehe die Debatten auf YouTube.com: http://www.youtube.com/ watch? $\mathrm{v}=\mathrm{MI} 4 \mathrm{q} 7 \mathrm{jSrgjo}$; http://www.youtube.com/watch? $\mathrm{v}=\mathrm{s} \mathrm{X}_{4} \mathrm{MG}$ 6_1X8w (Zugriff: 27.0I. 2016) 
wächst (Topalov, Jacobs 2008). Dieser Raum umfasst eine Summe von symbolischen und verbalen Konventionen und hat seine soziale Dimension.

Das erstreckt sich nicht nur auf den Prozess des individuellen Erinnerns, sondern auf das Tradieren des historischen Gedächtnisses insgesamt. Dieses wird zwar im Geschichtsunterricht und anderswo mit maximalem Anspruch auf Ganzheitlichkeit weitergegeben, allerdings erst nachdem ausgewählt wurde, was für unsere Gegenwart relevant ist. Das lässt sich gut auf die Realität der deutsch-tschechischen Beziehungen und die deutschtschechische Geschichte übertragen, für die eben diese Phänomene kennzeichnend sind. Die Wiederholung von Erinnerungen nennt Halbwachs Tradition und definiert sie als Umformung der Erinnerungen in eine bestimmte Gewohnheit. Halbwachs unterscheidet dabei streng zwischen individuellem und kollektivem Gedächtnis. Das individuelle Gedächtnis bildet sich bei jedem Individuum im Verlauf seiner Sozialisierung heraus. Halbwachs spricht von der Notwendigkeit kommunikativer Prozesse und Beziehungen, in die der Einzelne eintritt. Unsere Erinnerungen stützen sich demnach auf das, was wir in der Kommunikation mit anderen erfahren haben. Diese Erinnerungen passen sich unserer aktuellen Wahrnehmung an. Mit anderen Worten: Es gibt keine Erinnerung ohne Wahrnehmung und keine Wahrnehmung ohne Erinnerung. Die Gegenwart betrachten wir stets unter Einbeziehung der Vergangenheit, ganz gleich, ob sie selbst erlebt oder überliefert ist. Berücksichtigt werden muss jedoch, dass jeder Mensch anders wahrnimmt und erinnert, in anderen Zusammenhängen und mit anderen Akzenten (Alheit 1990). Die Erinnerung spiegelt die Position des Individuums im jeweiligen sozialen Umfeld wider. Jeder von uns hält sie für seine eigene, in Wirklichkeit ist sie jedoch beeinflusst vom sozialen Umfeld und dem Kollektiv, in dem wir leben und uns bewegen (Assmann 1999, 35). Darauf macht u. a. Krzystof Pomian in seinen Untersuchungen aufmerksam, indem er außer von nationalen Gedächtnisdebatten, die jeweils in einem spezifischen historisch-kulturellen Umfeld stattfinden, auch von einem europäischen Kollektivgedächtnis spricht (Pomian 1998). An das, was wir erlebt haben, erinnern wir uns in Gegenwart anderer, ganz gleich, ob sie tatsächlich oder nur fiktiv anwesend sind.

Das Konzept des kollektiven Gedächtnisses ist keinesfalls eindeutig und konsistent, und zwar vor allem in der psychologischen Dimension und der Interpretation der selektiven Beschaffenheit des Gedächtnisses. Es geht davon aus, dass es sich praktisch um die Rekonstruktion der Vergangenheit sozialer Gruppen handelt, also um die Adaption längst vergangener Tatsachen, und zwar so, dass sie den Überzeugungen und geistigen Bedürfnissen der Gegenwart entsprechen und eine Gruppenidentität ausdrücken (Pešková 1998). Das geschieht auf der Grundlage einer Fragmentierung des Erinnerns, wobei bestimmte geschichtliche Ereignisse aus unterschiedlichsten Gründen ausgelassen oder sogar tabuisiert werden. Die Beschäftigung mit dem historischen Gedächtnis sozialer Gruppen ist natürlich überaus wichtig, vor allem wegen der Funktion, die diese mentalen Operationen im Prozess der Formung und Stärkung der Identität haben (Lee/Yeoh 2005).

Der Begriff der Europäisierung bezieht sich auf die neue Wirklichkeit, die Europa vor allem in den letzten Jahren erlebt, und zwar besonders infolge der Annahme der letzten primärrechtlichen Verträge, des Vertrags von Nizza und des Vertrags von Lissabon. ${ }^{5}$ Sicher würde in diesem Zusammenhang auch die in den Referenden abgelehnte Europaverfassung hineinpassen. Europäisierung bedeutet im Grunde eine Art Globalisierung im Kleinen, ausgehend von den qualitativen Veränderungen am Ende des 2O. und zu Beginn des 2I. Jh., in deren Folge wir eine Bewegung hin zur neuen allgemeinen Reorganisation des europäischen Raumes beobachten können. Sie wird von den Institutionen der EU zur konsequenten Supranationalisierung geführt, die die Antwort auf Globalisierungstendenzen, aber auch auf die sich verringernde Integrationsbereitschaft einzelner Staaten ist. Sie mag zwar im Widerspruch zu den Wünschen eines Teils der politischen Spitzen der EU-Mitgliedsländer stehen, dennoch haben auch diese den Lissabon-Vertrag, der ja das höhere Maß an Integration verankert, mitunterzeichnet und so zu seiner Ratifizierung beigetragen. Borneman und Fowler sagen übrigens sehr direkt, dass die Europäisierung, also das schrittweise Sich-Durchsetzen der Europäischen Union zulasten der einzelnen Staaten, nie die Existenz von Nationalstaaten vollständig ersetzen können wird, dass die Europäisierung sich nie so weit durchsetzen lassen wird (Borneman/Fowler 1997). Die Neofunktionalisten verwenden allerdings trotzdem das Konzept der Europäisierung zur Erklärung der Realität der Europäischen Union und der europäischen Integration insofern, dass sie darin ein bedeutendes Moment der Erweiterung der Funktionen der EU und der Herausbildung einer gemeinsamen „europäischen" Identität suchen (Meyer 2004). Risse ist bspw. der Ansicht, dass die Agenda der Europäischen Union unausweichlich normaler Bestandteil der Politiken der Länder werden wird (Risse 20IO, 245). Hier bleibt allerdings die Frage offen, ob es angebracht ist, über das Individuum und den Staat europäische Interessen bzw. eine europäische Identität zu formulieren (Müller 20I2). Andererseits sind diese beiden die einzigen relevanten

5 Das Europäisierungskonzept wird in diesem Text in seiner politischen bzw. machtpolitischen Dimension betrachtet, wobei sein kultureller und gesellschaftlicher Aspekt etwas vernachlässigt wird (z. $B$. das wachsende Bewusstsein für gemeinsame Werte, Dialog- und Erinnerungskultur, das sich festigende Erleben einer friedlichen Nachbarschaft zwischen den Nationen) 
Subjekte, die glaubwürdige Träger einer solchen europäischen Identität sein können. Nach Olsen (2002) können wir insgesamt fünf mögliche Bedeutungen der Europäisierung feststellen. Das sind:

I. Veränderung und Erweiterung der territorialen $\mathrm{Au}$ ßengrenzen der EU

2. Weiterentwicklung der Institutionen und Organisationen auf europäischer Ebene

3. Vordringen europäischer Dimension in nationale und subnationale Institutionen

4. Export der europäischen politischen Organisation und Herrschaft in Länder außerhalb Europas

5. Unifizierung als politisches Projekt der Europäischen Union.

All dies scheint zu einem Verwischen der Unterschiede im Verständnis der Staaten zu führen. Das soll nicht heißen, dass die Staaten in den Augen ihrer Bürger an Kraft und Kompetenz verlieren, das wird sicher nicht der Fall sein, aber faktisch werden sie geschwächt, was die Ausübung der Staatsgewalt, die Entscheidungsgewalt über eine Reihe von Politiken usw. betrifft. Unter diesem Gesichtspunkt betrachtet ist die Europäisierung, also die Harmonisierung der unterschiedlichen Standpunkte und ihre positive Formulierung zum Zweck der Stärkung des "Kollektivgeistes“ der EU, auch in den deutsch-tschechischen Beziehungen offensichtlich (Novotný 20I3). Und es war ja auch die Europäische Union, die während der Beitrittsverhandlungen zur Beruhigung der Situation hinsichtlich einiger strittiger Punkte zwischen der Tschechischen Republik und ihren Nachbarn beigetragen hat: Im Fall Österreichs ging es um das KKW Temelín, aber es waren auch die deutsch-tschechischen bzw. österreichisch-tschechischen Beziehungen, konkret die Gültigkeit der Präsidialdekrete und deren Rolle im heutigen Rechtssystem Tschechiens. Hier war es vor allem das Gutachten Jochen Froweins, Professor am Max-Planck-Institut, der in seinem dreißigseitigen, als unabhängige Rechtsexpertise über die Auswirkungen der Dekrete auf die EU-Erweiterung erstellten Gutachten feststellte, dass diese Rechtsnormen kein Hindernis für den EU-Beitritt der Tschechischen Republik seien. Das Gutachten umging recht elegant ein Problem von grundsätzlicher Bedeutung, und zwar das Maß der Übereinstimmung der analysierten Dekrete mit der EULegislative. Im Gutachten ist allerdings der kritische Tonfall der Gutachter gegenüber diesen Rechtsnormen unverkennbar. ${ }^{6}$

In den Schlussfolgerungen der Analyse, die das Europäische Parlament im Frühjahr 2002 in Auftrag gegeben hatte, heißt es wörtlich: „Der Beitritt der Tschechischen Republik ist durch die Dekrete nicht gefährdet"

6 Am offenkundigsten ist dies bei Prof. Ulf Bernitz. http://www.europarl.europa.eu/RegData/etudes/etudes/join/2002/323934/DG-4AFET_ET\%282002\%29323934_CS.pdf (Zugriff: 27.01.2016)
(Frowein et al. 2002, 34 ff; European Commission 2002). $\mathrm{Zu}$ ähnlichen Feststellungen gelangten auch die Experten Ulf Bernitz und Lord Christopher Kingsland (ebd.). Nicht weniger wichtig waren die Rolle des Gutachtens, das die Europäische Kommission in Auftrag gegeben hatte, und die Vermittlerrolle G. Verheugens, der in der Tschechischen Republik eine öffentlich anerkannte und glaubwürdige Persönlichkeit wurde - zuerst bei den Verhandlungen zur Dt.-Tsch. Erklärung 1997, später als EUKommissar für die EU-Erweiterung.7 Das half damals selbstverständlich, die Stimmungswogen zu glätten. Zwar wurden Gegengutachten verfasst, die vom Bayerischen Landtag bzw. der Sudetendeutschen Landsmannschaft in Auftrag gegeben worden waren, es war jedoch Froweins Gutachten, das die Situation beruhigte und ein weiteres Problem in den Beziehungen zwischen Tschechen und Deutschen ausräumte.

\section{Die Debatten zwischen den Präsidentschafts- kandidaten Zeman und Schwarzenberg und die deutsch-tschechischen Themen}

Geschichte sollte nicht als Waffe eingesetzt werden, wie es in der Wahlkampagne der Präsidentschaftskandidaten, vor allem in der zweiten Runde, wiederholt geschah. Tatsächlich tauchte eine ganze Reihe interessanter Themen und Momente auf, in denen Geschichte eine wesentliche Rolle spielte und die Wählerpräferenzen der einzelnen Kandidaten gefestigt oder umgestaltet hat. Einer eigenen Untersuchung wert wären hier die Rollen, die der damalige Staatspräsidenten Václav Klaus, die kontroverse Anzeige in der meist gelesenen tschechischen Boulevard-Zeitung Blesk und die Vergangenheit des Schwiegervaters K. Schwarzenbergs (dies alles kam in der Kampagne zum Einsatz und betraf die deutschtschechischen Beziehungen) in diesem Wahlkampf gespielt haben. Ähnlich wie wir die Präsidentschaftswahl in zwei Wahlrunden gliedern, können wir auch das Heranziehen deutsch-tschechischer Geschichtsthemen durch diese beiden Wahlrunden begrenzen. Der besondere Anlass dazu dürfte die Stärkung der Position Karl Schwarzenbergs in den Wählerpräferenzen gewesen

7 Auch wenn es nicht Gegenstand dieses Textes ist, soll hier hervorgehoben werden, dass ebenso die Aufnahme Tschechiens in die NATO und damit de facto das "Ausräumen“ von Sicherheitsbedenken gegenüber Deutschland eine außerordentliche Rolle bei der Schaffung eines vertrauenswürdigen Images der BRD gespielt hat. Die BRD wurde viel stärker als Verbündeter wahrgenommen, der darüber hinaus militärpolitisch zurückhaltend agierte (siehe die Bereitschaft der BRD, sich der amerikanischen Politik bei der Invasion im Irak zu widersetzen). Eine weitere Dimension, die eindeutig zur Verbesserung der gegenseitigen deutsch-tschechischen Beziehungen beigetragen hat, ist das Entstehen eines dichten Netzwerks bilateraler Beziehungen, die dezentralisiert sind, alle Bereiche des gesellschaftlichen Lebens umfassen und überwiegend unabhängig von der Politik sind. (Novotný 20I3) 
sein, aber auch die Art und Weise, wie seine Kampagne insgesamt geführt wurde, und ihre wirksame Steigerung. Während im Wahlkampf vor der ersten Wahlrunde faktisch keine erkennbare Instrumentalisierung deutsch-tschechischer Themen erkennbar war, was vor allem auf die Orientierung Miloš Zemans auf den künftigen Gegenkandidaten Jan Fischer zurückgeführt werden kann, änderte sich die Situation in der zweiten Wahlrunde.

Sicher spielte in diesem Zusammenhang die Tatsache eine Rolle, dass dem für seine kritischen Äußerungen über Sudetendeutsche vor allem aus seiner Zeit als Ministerpräsident bekannten Miloš Zeman als Gegenkandidat nun der Vorsitzende von TOP o9, stellv. Ministerpräsident der Regierung und Außenminister Karl Schwarzenberg gegenüberstand. Andernfalls (bspw. in Fernsehduellen Zeman vs. Fischer) hätten deutschtschechische Geschichtsthemen im Wahlkampf keine solche Relevanz gehabt. Von entscheidender Bedeutung war dabei eben nicht der Wahlkampf als solcher, weder Outdoorkampagne oder andere Formen persönlicher Begegnungen, Verteilung von Handzetteln usw. sondern es waren ausschließlich die Fernsehdebatten, ergänzt von Rundfunkauftritten beider Kandidaten. Im Wahlkampf selbst haben neben den bereits erwähnten Kampagnen die Fernsehauftritte der Kandidaten eine wesentliche Rolle gespielt. Gegenstand der Analyse sind jedoch nur die Fernsehdebatten, in denen Fragen aus dem Themenkreis der deutsch-tschechischen Beziehungen aufgeworfen wurden. Ausgeklammert werden Diskussionen, die in anderen Medien stattfanden. Einerseits sind die Fernsehdebatten für den Verlauf der zweiten Runde essenziell vor allem wegen der Macht des Fernsehens als audiovisuelles Medium, andererseits waren es gerade die Fernsehdebatten, bei denen für ein Streitgespräch die absolut meiste Zeit zur Verfügung stand.

\section{Erste Fernsehdebatte zwischen den Präsident- schaftskandidaten im Tschechischen Fernsehen und auf TV Prima}

Den Auftakt machte das zweistündige TV-Duell zwischen den Präsidentschaftskandidaten Zeman und Schwarzenberg am 17. Januar $2013 \mathrm{im}$ Tschechischen Fernsehen, mehr als eine Woche vor der zweiten Runde der Präsidentschaftswahl. Gesendet wurde aus dem Hybernia-Theater (ebenso wie eine Woche später) und es war Moderator Václav Moravec, der im zweiten Teil der Debatte den beiden Kandidaten die Frage stellte, die - wie er sagte - aus zahlreichen Zuschaueranfragen hervorgegangen war: Wie würden sie sich in dem hypothetischen Fall verhalten, dass Regierung und Parlament überein- kämen, die Gültigkeit der „Beneš-Dekrete“ ${ }^{\text {" }}$ aufzuheben, und ob sie als Staatspräsident an der Aufhebung der Dekrete mitwirken würden oder nicht. An dieser Stelle erklang der entscheidende Satz Karl Schwarzenbergs, der die nachfolgende Debatte zu den deutsch-tschechischen Themen entzündete, nämlich, dass „die Beneš-Dekrete schon seit 20 Jahren nicht mehr gelten“. Seine Argumentation basierte auf der Behauptung, mit der Aufnahme der Charta der Grundrechte und -freiheiten in die tschechische Verfassung sei „die Gültigkeit der Beneš-Dekrete beendet". Es handele sich also um die Ungültigkeit der Dekrete ab dem Zeitpunkt der Wirksamkeit dieser Charta. Schwarzenberg bekräftigte sein Argument, indem er sagte, „was bereits aufgehoben ist, könne nicht noch einmal aufgehoben werden“. Das war ein wesentlicher „Auslöser“ für Miloš Zeman, der Schwarzenberg auf den Unterschied zwischen Dekreten und Gesetzen aufmerksam machte und bemerkte, als Minister dieses Landes sollte er diese beiden Arten von Dokumenten nicht miteinander verwechseln, und weiter, dass laut Feststellung des (tschechischen) Verfassungsgerichts diese Dekrete, die zu Gesetzen wurden, nachdem sie von der ersten Nachkriegs-Nationalversammlung als solche gebilligt worden waren, gültig und ein Bestandteil der Rechtsordnung der Tschechischen Republik seien. Zeman ging dann mit Belegen aus der „autorisierten Biographie Schwarzenbergs" in die Offensive und stellte diesem die Frage, ob es wahr sei, dass Schwarzenberg dem damaligen Staatspräsidenten Václav Havel geraten habe, sich bei den Sudetendeutschen zu entschuldigen. ${ }^{9}$ Weiter fragte er, ob die Behauptung eines anderen Biographen (aus Österreich) - diesmal nicht autorisiert - wahr sei, der Minister habe früher einmal vom Anspruch der Sudetendeutschen auf Rückgabe der Staatsbürgerschaft und auch des Eigentums gesprochen. Karl Schwarzenberg machte Zeman darauf aufmerksam, dass er ihn falsch zitiert habe, er verwies allerdings darauf, dass „das, was wir nach 1945 getan haben, heute als grobe Verletzung der Menschenrechte verurteilt werden würde und dass sich wahrscheinlich die damalige Regierung samt dem Staatspräsidenten in Den Haag wiederfinden würde“. Dafür erhielt Schwarzenberg mäßigen Beifall aus dem Publikum. Zeman antwortete: „Ihnen

8 Der Begriff wurde in dieser Form in der Wahlkampagne völlig wertneutral verwendet.

9 Bereits bei seiner ersten Auslandsreise nach der Wahl zum Staatspräsidenten besuchte Havel beide deutschen Staaten. Er äußerte dort - trotz der skeptischen öffentlichen Meinung in der Heimat Zustimmung zur Vereinigung, vorausgesetzt, sie würde im gesamteuropäischen Konsens vollzogen. Vor allem in den Grenzgebieten häuften sich Befürchtungen, eine kritische Bewältigung der historischen Ereignisse könnte Reparationen, Besitzrückgabe oder gar die erneute Abtretung der Sudetengebiete evozieren. Bereits vor diesem Staatsbesuch hatte Havel die Vertreibung der Deutschen nach 1945 verurteilt. Schwarzenberg leitete als Kanzler während der Amtszeit Havels zwei Jahre lang das Büro des Staatspräsidenten (Novotný 2015). 
zufolge würde also Beneš als Kriegsverbrecher verurteilt werden. Nichts anderes haben Sie jetzt gesagt." Schwarzenberg half sich mit den Argumenten V. Havels von der „Ansteckung mit dem Nazi-Bazillus, als wir die Mitbürger, auch die, die unschuldig waren, von hier vertrieben haben". Ihm zufolge hatten viele Deutsche die Republik nicht verraten, sie seien zu Unrecht abgeschoben worden. Schwarzenberg verwies im Weiteren auf die Verfolgung der Sudetendeutschen während der NS-Zeit und ihren Widerstand gegen den Nationalsozialismus und warf der damaligen Staatsführung die Anwendung des Prinzips der Kollektivschuld vor. „Wir haben damals tatsächlich schwer gefehlt und dabei bleibe ich."

Diese Worte Schwarzenbergs hatten ein Nachspiel in der Debatte der beiden Präsidentschaftskandidaten auf TV Prima, die am darauffolgenden Tag stattfand. Hier gab Schwarzenberg zu, er habe sich im Zusammenhang mit der Gültigkeit der Dekrete ungenau ausgedrückt, und sagte, die Dekrete haben ihre Wirksamkeit verloren. „Gültig sind sie, sie sind Bestandteil der geltenden Rechtsordnung“, sagte der Minister wörtlich. Zeman erwiderte, gerade wegen der skandalösen Äußerungen Schwarzenbergs trage er am Revers seines Sakkos die Anstecknadel mit der tschechischen Staatsflagge, und erinnerte daran, dass in der Debatte im Tschechischen Fernsehen Karl Schwarzenberg Beneš als Kriegsverbrecher bezeichnet habe, der vor das Haager Tribunal gehöre. Schwarzenberg versuchte sich zu rechtfertigen, indem er ausführte, dass ähnliche Handlungen, wie sie im tschechoslowakischen Grenzgebiet nach dem Krieg begangen wurden, heute die Verurteilung der Repräsentanten solcher Gewaltakte und ihre Vorladung vor das Strafgericht nach sich ziehen würden. Zeman konterte, Schwarzenberg habe tatsächlich Beneš namentlich genannt und halte diesen für einen Verbrecher. „Herr Minister, bisher hätte ich absolut respektiert, wenn Sie Staatspräsident geworden wären, aber wer einen Präsidenten der Tschechoslowakei als Kriegsverbrecher bezeichnet - den Beweis dafür haben wir in der Aufzeichnung - spricht wie ein Sudetendeutscher und nicht wie der Staatspräsident", sagte Zeman wörtlich. Hier erwies sich M. Zeman als hervorragender Rhetoriker, der die Diskussion unter Kontrolle hat und der mit der Erinnerung an den Ausspruch, Edvard Beneš sei einem Kriegsverbrecher gleichzusetzen, Schwarzenberg in die Defensive drängt. Die Thematik Dekrete und Bewertung des ehemaligen Präsidenten Beneš gelangte so deutlich stärker in den öffentlichen Raum und damit auch in die Wahlkampfdebatten. Während ihr in der Sendung des Tschechischen Fernsehens kaum mehr als fünf Minuten gewidmet wurden, waren es in der Debatte auf TV Prima bereits I2 Minuten.

\section{Zweite Fernsehdebatte zwischen den Präsident- schaftskandidaten im Tschechischen Fernsehen}

Die zweite Debatte fand im Tschechischen Fernsehen genau eine Woche später, am 24. Januar, am gleichen Ort wie die erste statt. Ihr Verlauf wurde stark beeinflusst von Fragen zu den Themen Sudetendeutsche und deutsch-tschechische Beziehungen allgemein. Mittelpunkt der Diskussion waren auch weiterhin vor allem die Bezeichnung Edvard Benešs als Kriegsverbrecher und die Gültigkeit der Beneš-Dekrete. Gesprächsleiter Václav Moravec stellte zu Beginn fest, das Thema BenešDekrete beherrsche seit der letzten Woche den Wahlkampf, und stellte Karl Schwarzenberg bereits in der zehnten Minute der Debatte - was auf die Dringlichkeit und Aktualität dieser Themen hindeutet - die Frage, ob seine Worte die deutsch-tschechischen Beziehungen nicht in den Zustand vor der Deutsch-Tschechischen Erklärung zurückversetzten. Das bestritt der Minister. Miloš Zeman sagte zuerst, die Vertreibung sei im Einklang mit der Potsdamer Konferenz gewesen. Die Redaktion des Tschechischen Fernsehens hatte allerdings die Rede vom 25.IO.I990 vor dem Föderalen Parlament aus dem Archiv geholt, in der Zeman über die Verletzung der Demokratie nach 1945 spricht und sagt, der Transfer der deutschen Bevölkerung sei „genau im Geiste Stalin'scher Zwangsumsiedlungen" verlaufen. In der Debatte im Studio korrigierte Zeman dies dahingehend, er habe damals vor dem Parlament von der wilden Vertreibung gesprochen, die vor dem Potsdamer Abkommen stattgefunden hatte. „Die wilde Vertreibung war unmoralisch", sagte er und zitierte Artikel I3 des Potsdamer Abkommens, der besagt, der Transfer sei human durchzuführen. Dann ging er in die Offensive und sagte, Karl Schwarzenberg dürfte mit seinen Worten von der Ungültigkeit der Dekrete gewissen politischen Kreisen in Österreich und Deutschland (in dieser Reihenfolge) Freude bereitet haben. Er ging noch weiter, indem er sagte, Minister Schwarzenberg habe seiner Ansicht nach „unsere Beziehungen zur Slowakei“ geschädigt. Er berief sich dabei auf (den slowakischen) Premierminister Robert Fico, der sein Befremden darüber geäußert hatte, dass ein tschechischer Außenminister die BenešDekrete in Frage stellt. Im weiteren Verlauf erwähnte Zeman dann die Kritik des slowakischen konservativen Politikers Ján Čarnogurský, der die Aussage des tschechischen Außenministers ebenfalls mit Verwunderung registriert hatte.

In der Debatte kam auch die Reaktion des Sprechers der Sudetendeutschen Volksgruppe und Vorsitzenden der Sudetendeutschen Landsmannschaft Bernd Posselt zur Sprache. Dieser war bereits eine Woche zuvor in der Fernsehdebatte im Tschechischen Fernsehen erwähnt worden, da er sich - Zeman zufolge - in der Süddeutschen Zeitung über den Erfolg Karl Schwarzenbergs 
beifällig geäußert haben sollte. Im Tschechischen Fernsehen wurde Posselts Aussage zitiert, er habe in der Zeit des Präsidentschafts-Wahlkampfes nicht eine einzige Presseerklärung abgegeben. Damit war offensichtlich, dass dieses Argument nicht auf Wahrheit beruhte. Zeman hatte sich bereits einen Tag zuvor in der Rundfunksendung von Radio Impulz für seinen Irrtum entschuldigt, in seinem Fernsehauftritt wiederholte er jedoch gegenüber Karl Schwarzenberg seine Entschuldigung nicht (er war sich sicher bewusst, welche Macht gerade das Fernsehen darstellt und dass diese Unwahrheit in der öffentlichen Diskussion weiter Resonanz finden und sie zu seinem Vorteil beeinflussen kann). Zeman selbst erklärte seinen Irrtum mit dem Hinweis auf eine entsprechende Nachricht in der Internettageszeitung Britské listy (Britische Blätter), er sagte sogar, er halte die Journalisten von Britské listy für glaubwürdig. Zeman hatte demnach nicht an der Quelle recherchiert. Ein so großes Vertrauen in die Arbeit der Journalisten ist, vor allem wenn man Zemans Einstellung gegenüber Journalisten kennt, recht verwunderlich.

Václav Moravec stellte abschließend in diesem Fragen-Block, der de facto der erste Themenblock dieser Debatte war, beiden Kandidaten die Frage, ob es überhaupt notwendig sei, dieses Thema im Wahlkampf und in den Diskussionen zur Präsidentschaftswahl aufzugreifen. Dabei war er es gewesen, der mit seiner Frage nach der Gültigkeit der Dekrete dieses Thema in den Wahlkampf gebracht hatte. Karl Schwarzenberg antwortete darauf, er wünsche sich, die Debatte würde über aktuelle Themen geführt, und sagte noch einmal, die Aussagen Miloš Zemans seien falsch. Wörtlich sagte er: „Auch hier haben Sie etwas behauptet, was ich nie gesagt habe. Ich habe die Beneš-Dekrete nicht infrage gestellt. Ich habe nie von Genozid gesprochen. Nie habe ich dergleichen gesagt, aber Sie haben es behauptet. Sie wollten die tschechische Bevölkerung in den grenznahen Gebieten ganz bewusst verunsichern." Zeman konterte mit dem lange vor der Debatte veröffentlichten Interview in der Tageszeitung MF Dnes, in dem Schwarzenberg die Dekrete als Schande für die tschechische Geschichte bezeichnet hatte. Schwarzenberg erwiderte darauf, dass „die Aussiedlung eines Drittels unserer Bevölkerung kein Ruhmesblatt für uns ist". Damit war dieser Teil der Debatte beendet und man widmete sich weiteren Fragen und Problemen. Am Schluss, als den Kandidaten aus dem $\mathrm{Pu}$ blikum Fragen gestellt werden durften, gab es noch eine Frage zur deutsch-tschechischen Geschichte. Im Lager Miloš Zemans befand sich Professor Jan Kuklík von der Juristischen Fakultät der Karls-Universität, der Schwarzenberg nach Alternativen zur Vertreibung fragte. Die weitere Diskussion verlief dann ruhig und konfliktfrei.

\section{Die Dekrete, E. Beneš und die Vertreibung aus Sicht der tschechischen Öffentlichkeit}

Die in den einzelnen Debatten zwischen den Präsidentschaftskandidaten im Mittelpunkt stehenden „sudetendeutschen" Themen waren:

- die Person Edvard Benešs

- Standpunkt zur Vertreibung der Deutschen

- Standpunkt zu den Präsidialdekreten

In den Debatten kam dann zum Ausdruck, was bereits seit langem in der tschechischen Öffentlichkeit als Thema wahrgenommen wird, was Spannungen und besonders bei der älteren Generation auch erhebliche Emotionen hervorruft. Vor allem muss gesagt werden, dass die Dekrete des tschechoslowakischen Staatspräsidenten Edvard Beneš im kollektiven Geschichtsgedächtnis der Öffentlichkeit eine wesentliche Rolle spielen, ebenso die Person Edvard Benešs. Erinnert sei hier an die Gültigkeit der „Lex Beneš“ (Nr. 292/2004), deren einziger Paragraf lautet: Edvard Beneš hat sich um den Staat verdient gemacht. Das deutet auf eine erhebliche Selektivität im historischen Gedächtnis hin, auf die bereits u. a. Halbwachs verweist. Beneš gehört heute tatsächlich zu den Persönlichkeiten der tschechoslowakischen Geschichte, die von der Öffentlichkeit zwar widersprüchlich, aber durchaus - mit Rücksicht auf die tragischen Zeitumstände seiner Präsidentschaft - positiv wahrgenommen werden. Das resultiert aus seinem Wirken an der Seite T. G. Masaryks in der sog. ersten tschechoslowakischen Republik, die im tschechischen historischen Denken sehr positiv wahrgenommen wird, und aus seiner Tätigkeit im tschechoslowakischen Exil, wo er die "Seele" der Organisation für die Koordinierung der Aktivitäten des einheimischen Widerstands und des Exilwiderstands war, und hängt auch mit den Dekreten zusammen (Novotný 2009). Die Unantastbarkeit der Dekrete, die in journalistischer Abkürzung seinen Namen tragen, wird so betrachtet verständlich, und die Notwendigkeit ihrer Gültigkeit wird auch von einem erheblichen Teil der tschechischen Konservativen und von Geschichtswissenschaftlern betont (Pavlíček et al. 2002; Dejmek et al. 1999). Die Ergebnisse der Meinungsumfragen - vor allem vom $\mathrm{CVVM}^{10}$ - belegen langfristig den mehrheitlichen Wunsch in der tschechischen Gesellschaft, die Dekrete mögen ihre Gültigkeit behalten. In der bisher letzten Umfrage hatte sich fast die Hälfte der Befragten

IO $\mathrm{Zu}$ den Befragungen und deren Parametern siehe Novotný 2012. Dieser Aufsatz bezieht sich vor allem auf die landesweite Befragung, die vom CVVM (Meinungsforschungszentrum des Soziologischen Instituts der Akademie der Wissenschaften der Tschechischen Republik) im November 2012 und 2014 mit jeweils IOoo Befragten durchgeführt wurde, und berücksichtigt auch Vergleiche mit früher durchgeführten Untersuchungen. Hauptthemen sind hierbei die Sicht der tschechischen Öffentlichkeit auf die eigentlichen Dekrete und die Vertreibung der Sudetendeutschen. 
(49\%) dafür ausgesprochen. ${ }^{\text {II }}$ Sie tendiert zu der Auffassung, die Beneš-Dekrete sollten auch weiterhin gelten. Man sieht hier eine bemerkenswerte emotionale Bindung an die Dekrete, die als "Sieg“ im deutsch-tschechischen und österreichisch-tschechischen Vergangenheitsdiskurs wahrgenommen werden. Hingegen meinen I7 \%, die Dekrete sollten aufgehoben werden und mehr als ein Drittel der Befragten (34 \%) konnte keine Aussage zu dieser Problematik machen. Annähernd zwei Fünftel der tschechischen Bevölkerung ( 42 \%) halten die Vertreibung für gerecht, demgegenüber wird sie von insgesamt 39 \% für ungerecht gehalten.

Ähnlich wie bei den Dekreten sind leichte Veränderungen auch in der Frage nach der Gerechtigkeit der Dekrete zu verzeichnen. Das zeigt sich vor allem darin, dass in den zurückliegenden Jahren die Zahl der Befürworter der Vertreibung allmählich zurückgegangen ist, also jener, die die Aussiedlung als gerechten Akt werten, während die Zahl derer wächst, die diesen Akt für ungerecht halten (und das insgesamt, d. h. in der Summe aller Positionen, in denen von der Vertreibung als „ungerecht" gesprochen wird, sowie im Rahmen der einzelnen Positionen) (Novotný 2012). Auch die Entwicklung bei der Frage nach der "Ungerechtigkeit der Dekrete“ zeigt eine gewisse Dynamik - in den letzten zehn Jahren ist der Anteil der Antworten, die Dekrete seien ungerecht, um rund to \% gestiegen. Sehr unterschiedlich sind die Ansichten darüber, ob die Vertreibung ein gerechter oder ungerechter Akt war, bei den verschiedenen Altersgruppen. Hier spielt das eigene Erleben, die Weitergabe von Wissen in der Familie und in der Schule und allgemein auch der zeitliche Abstand zu dem analysierten Ereignis eine Rolle. In der Altersgruppe über 60 Jahre hält fast die Hälfte der Befragten (49\%) die Vertreibung für gerechtfertigt. Bei jüngeren Leuten unter 30 Jahren sind nur 32 \% dieser Meinung. Dieses Ergebnis ist sicher auch von der Tatsache beeinflusst, dass sich junge Menschen, im Alter zwischen I5 und 29 Jahren, nicht sehr für das Thema Vertreibung der Sudetendeutschen interessieren bzw. dass sie keine eindeutige Meinung zu diesem historischen Ereignis haben (36 \%). Mit anderen Worten: In ihrem historischen Gedächtnis fehlen das eigene Erleben und auch die Propaganda während der kommunistischen Ära.

\section{Schluss}

Am Anfang des Artikels wurde die Hypothese aufgestellt, dass - ungeachtet aller Aufmerksamkeit, die Themen wie Sudetendeutsche, Vertreibung der Deutschen aus der Tschechoslowakei und das Wirken Edvard

II http://cvvm.soc.cas.cz/media/com_form2content/documents/cI/ a6665/f3/pmi2orio.pdf (Zugriff 27.0I. 20I6)
Benešs im Rahmen der bilateralen Beziehungen verdienen - die Ergebnisse der Präsidentschaftswahl von diesen Themen nicht wesentlich beeinflusst wurden. Diese Auffassung sieht der Autor als bestätigt an, auch wenn das kontrovers klingen mag und widersprechende Meinungen hervorruft. Hier ist sich der Verfasser durchaus bewusst, dass z. B. einige Meinungsforschungsinstitutebesonders deutlich wird das u. a. bei der Agentur Median und ihrer Umfrage (Median 2013) ${ }^{12}$ - zu der Feststellung gelangten, dass die deutsch-tschechischen Beziehungen eine stark determinierende Rolle gespielt haben, jedoch das Wahlergebnis durch sie nicht wesentlich beeinflusst wurde, denn 45 Prozent der Stimmen für den Kandidaten Schwarzenberg bedeuteten für diesen keine eklatante Niederlage. Die deutsch-tschechischen Beziehungen waren also für die Wahl im Grunde relevant, die Debatte hatte einen rein tschechisch-tschechischen Charakter. Versuchen wir uns einmal vorzustellen, wie Ende des vergangenen Jahrhunderts, als es heftige Streitigkeiten zwischen Deutschen und Tschechen gab, eine Wahl nach derart hektischen Debatten über Präsidialdekrete und Vertreibung ausgegangen wäre. Ein Kandidat mit den Ansichten Schwarzenbergs wäre bei dieser Wahl definitiv durchgefallen, so emotional stark war das Thema im kollektiven Bewusstsein verankert.

Dafür, dass deutsch-tschechische Themen auf die Wahl selbst und vor allem auf das Wahlergebnis keinen wesentlichen Einfluss hatten, sprechen vor allem folgende Argumente:

Erstens galt Miloš Zeman verschiedenen Meinungsumfragen zufolge bereits vor der zweiten Wahlrunde als der Wahlsieger und das bestätigte auch das tatsächliche Wahlergebnis, das keinesfalls so ausgeglichen erwartet wurde, wie es dann in der zweiten Wahlrunde tatsächlich war. Die oben genannten Themen mit Bezug zum deutsch-tschechischen Verhältnis haben Miloš Zeman eindeutig nicht $\mathrm{zu}$ mehr Prozentpunkten verholfen. Vielmehr wurde die Position Schwarzenbergs leicht gestärkt, wie ersichtlich wird, wenn man die Wahlergebnisse mit den Prognosen aus der Zeit vor der Wahl vergleicht (Maškarinec 2013).

Nun ist bei Wahlen, die aus zwei Runden bestehen, schwer einzuschätzen, wer letztendlich die meisten Wähler für sich gewinnt. Während des Wahlkampfs waren es eindeutig andere Themen, die das Ergebnis erhärteten. Miloš Zeman war ein beliebter linksgerichteter

I2 Der häufigste Grund für den Wechsel der Wähler von M. Zeman (Nichtwähler oder Stimme für K. Schwarzenberg) war der Agentur Median zufolge die politische Vergangenheit Zemans und seine „Fälle“. Die „Abtrünnigen“ K. Schwarzenbergs (Nichtwähler oder Stimme für M. Zeman) waren der Umfrage zufolge von den Diskussionen über die Beneš-Dekrete, der Beteiligung des Kandidaten an der Nečas-Regierung und den Debatten zwischen den Präsidentschaftskandidaten wesentlich beeinflusst worden. Angesichts der 45 \% Wählerstimmen für Schwarzenberg im Endergebnis dürften der Verlust der Wählergunst und der Wechsel der Wähler zum Gegenkandidaten gering gewesen sein. 
Politiker und früherer Ministerpräsident, der auch während seiner Zeit als „Pensionist" ein aktiver und witziger Glossator des politischen Geschehens in Tschechien war, was ihm dauerhafte Sympathien bei einem nicht geringen Teil der tschechischen Bevölkerung sicherte. Die Präsidentschaftswahl fand zu einem für ihn günstigen Zeitpunkt statt - in der Gesellschaft herrschte eine gewisse Müdigkeit gegenüber konservativen Regierungen, die Zeman ebenfalls laut kritisierte. Es war eine für Zeman langfristig positive Gesamtlage, die ihm die Unterstützung bestimmter Gesellschaftsschichten sicherte, und daran änderte sich auch während des Wahlkampfs kaum etwas.

Karl Schwarzenberg war dagegen ein ganz anderer Präsidentschaftskandidaten-Typus: Seine Ausgangsposition war nicht günstig, er war Minister in einer unpopulären Regierung, die mit einer Reihe von Skandalen zu kämpfen hatte. Erst im Verlauf des Wahlkampfs begannen sich seine Wählerpräferenzen in die andere Richtung zu entwickeln. Während sie bei M. Zeman von Anfang an hoch und konstant waren, wuchsen bei K. Schwarzenberg diese erst mit der Wahlkampagne selbst. Ohne diese Wahlkampagne hätte Schwarzenberg als Präsidentschaftskandidat Platz vier belegt. So war es ihm gelungen, mit seiner Kampagne neue Wählergruppen anzusprechen und für sich zu gewinnen, vor allem die jüngere Generation. Das reichte jedoch nicht aus, um die starke Position Miloš Zemans zu erschüttern.

Man muss sich natürlich vergegenwärtigen, dass es schwierig ist, den Einfluss deutsch-tschechischer Themen auf das Wahlergebnis nachzuweisen. Untersucht wurde hier der Einfluss eines isolierten Themas und das Maß seines Einflusses auf das Wahlergebnis, was kompliziert und anhand des Wahlergebnisses schwierig zu analysieren ist. Klarerweise wurde das Wahlergebnis von einer Anzahl weiterer Faktoren beeinflusst. Das Ergebnis der zweiten Wahlrunde war allerdings relativ ausgeglichen und die Ursache dafür war - neben anderen Aspekten - mit Sicherheit auch die geringere Relevanz deutsch-tschechischer Geschichtsthemen im öffentlichen Diskurs. Andernfalls hätten diese Themen das Ergebnis wesentlich rasanter in Richtung Wahlsieg M. Zemans ausschlagen lassen.

Ein zweites Argument ist, dass sich heute vor allem bei dem jüngeren Teil der tschechischen Bevölkerung eine gewisse Veränderung in der Sichtweise auf die Sudetendeutschen und die Vertreibung zeigt. Die Zahl derer, die die Vertreibung für einen gerechtfertigten Nachkriegsakt halten, geht zurück. Einen gewissen Anteil daran hat - neben der oben beschriebenen Europäisierung - u. a. auch das zeitgenössische tschechische Filmschaffen, das mit Filmen, in denen das Leid und erlittene Unrecht der Deutschen dargestellt wird (z. B. in dem Film Habermanns Mühle aus dem Jahr 20IO), diese von einer anderen Seite bzw. generell vielschichtiger zeigt und nicht nur die Tatsache interpretiert, dass sie sich mit Hitler "eingelassen" und mit dessen Weltanschauung sympathisiert haben. Das wird notwendig zu einer Neudefinierung der Vertreibung und deren Rolle im Geschichtsgedächtnis eines beträchtlichen Teils der tschechischen Bevölkerung und damit auch in der Realität des Zusammenlebens von Tschechen und Deutschen bei Teilen der jüngeren Generation führen.

Das wurde auch bei den Fernsehdebatten zwischen den Präsidentschaftskandidaten deutlich, als ein nicht geringer Teil des Publikums im Saal Karl Schwarzenberg für seine kritischen Äußerungen zu den Beneš-Dekreten und der Vertreibung Beifall spendete. Das wiederum rief den damaligen Staatspräsidenten Václav Klaus auf den Plan, der die Aussagen Schwarzenbergs strikt ablehnte, vor allem die Relativierung der Beneš-Dekrete. Es wäre einer eingehenderen Analyse wert, wie sich diese aktuellen Momente des deutsch-tschechischen Dialogs im tschechischen Geschichtsgedächtnis widerspiegeln und wie dies bei Teilen der Bevölkerung zur Neudefinierung ihrer Haltung gegenüber der Vertreibung der Deutschen führen kann. Ein eigenständiges Thema wäre auch, wie sich diese Tendenzen auf die Politik auswirken.

Drittens kann als ein Paradox angesehen werden, dass Miloš Zeman mit der Art und Weise, wie er gewisses Unterlagenmaterial im Wahlkampf einsetzte, sich selbst hätte mehr schaden als nutzen können. Erwähnt sei hier nur der nicht existierende Artikel in der Süddeutschen Zeitung über die angebliche Unterstützung des Sprechers der sudetendeutschen Volksgruppe Bernd Posselt für den Präsidentschaftskandidaten Karl Schwarzenberg.

So erwies sich die Präsidentschaftswahl vielmehr als ein Zensus über das Fortbestehen der Regierung Petr Nečas und signalisierte eine mehrheitlich links gestimmte Gesellschaft, als dass sich in der Wahl die Konfliktgewalt der Themen der deutsch-tschechischen Beziehungen gezeigt hätte, die das Gesamtergebnis viel stärker beeinflusst haben würden. Die Argumentation des Präsidentschaftskandidaten Zeman legitimierte jedoch in gewisser Hinsicht die defensiv-konservativ-völkischen Töne.

Bezieht man die Themen der deutsch-tschechischen Beziehungen und ihre Verwendung im Wahlkampf um die Präsidentschaft auf die zwei Theorie-Bereiche, die im theoretischen Teil dieser Studie genannt wurden, d. h. auf das Konzept des Geschichtsgedächtnisses einerseits und die Auffassung von Europäisierung andererseits, dann erkennt man, dass vor allem die Präsidialdekrete und die Vertreibung der Deutschen aus der Tschechoslowakei auch weiterhin ein lebendiger Bestandteil des kollektiven Geschichtsgedächtnisses in der Tschechischen Republik sind.

Es zeigt sich immer wieder, dass die deutsch-tschechischen Beziehungen einerseits ein dankbares Thema 
in der öffentlichen Diskussion sind, es aber auch gelingt, Beruhigung in diese Diskussion zu bringen und dem Thema seine Brisanz zu nehmen. Ja, wir können sagen, dass sein Konfliktpotenzial die Tendenz hat zu erlöschen und dass es ganz entschieden nicht zunimmt. Der Grund dafür liegt nach Auffassung des Autors vor allem in der Europäisierung der gegenseitigen Beziehungen. Diese wird vor allem im Zusammenhang mit dem Abgeben "nationaler" Kompetenzen an die supranationale Ebene der Europäischen Union nicht immer als positiver Prozess gesehen. Aber herauszufinden, ob das so ist oder nicht, mag Gegenstand anderer Studien sein.

Das, was faktisch zur Entspannung und Beruhigung führte, war die Beilegung des Streits um die Kompatibilität der Präsidialdekrete mit dem europäischen Recht während des tschechischen Beitrittsprozesses. Heute ist es so, dass weder Prag noch Berlin eine Eskalation der gegenseitigen Beziehungen wünscht, dass sie bemüht sind, ihre Beziehungen auf kooperativer Ebene umfassend weiterzuentwickeln und dass diese Beziehungen die besten in ihrer bisherigen Geschichte sind. Weitere Verbesserungen dürften nicht allzu schwierig sein, da das Verhältnis zueinander in der Vergangenheit bis auf Ausnahmen eher schlecht war. Erfreulich ist allerdings die Tendenz zu wirklicher Normalisierung und weiterer Vertiefung der gegenseitigen Beziehungen. Und diese Entwicklung ist gut und richtig, trotz gelegentlicher „Pannen“ wie der beim größten innenpolitischen Ereignis des Jahres 2013 in der Tschechischen Republik, der Präsidentschaftswahl und der Wahlkampagne zuvor.

\section{Literatur}

Alheit, Peter (1990). Biographieforschung: eine Zwischenbilanz in der deutschen Soziologie, Bremen.

Assmann, Jan (1999). Das kulturelle Gedächtnis. Schrift, Erinnerung und politische Identität in frühen Hochkulturen, München.

Borneman, John/Nick Fowler (1997). Europeanization, in: Annual Review of Anthropology, Vol. 26(I), 482-5I5.

Coudenhove-Kalergi, Barbara/Oliver Rathkolb (Hg.). (2OI3). Die Benes-Dekrete, Wien.

Egger, Stephan (2003). Maurice Halbwachs. Aspekte des Werks, Konstanz.

European Commission (2002). The Czechoslovak Presidential Decrees in the light of acquis communautaire summary Cindiny of the Commission Services, Brussels.

Frowein, Jochen (2002). Legal Opinion on the BenešDecrees and the accession of the Czech Republic to the European Union, Brussels.

Le Goff, Jacques (1992). Geschichte und Gedächtnis. Historische Studien Bd. 6, Frankfurt am Main.
Goffman, Erwin (1974). Frame analysis: an essay on the organization of experience, New York.

Halbwachs, Maurice (1992). On Collective Memory (Heritage of Sociology), Chicago.

Houžvička, Václav (2015). Czech and Germans I848-2004. The Sudeten Question and the Transformation of Central Europe, Prag.

Kreisslová, Sandra (2013). Konstrukce etnické identity a kolektivní paměti v biografických vyprávěních českých Němců. Na př́kladu vzpomínek Němců na Chomutovsku. [Konstruktion der ethnischen Identität und des kollektiven Gedächtnisses in biographischen Erzählungen böhmischer Deutscher. Am Beispiel von Erinnerungen Deutscher in der Region Komotau], Prag.

Lee, Yong-Sock/Brenda Yeoh (Hg.) (2005). Globalisation and the Politics of Forgetting, London.

Maškarinec, Pavel (20I3). Prostorová analýza prezidentských volebv České republice v roce 20I3 [Raumanalyse der Präsidentschaftswahlen in der Tschechischen Republik 20I3], in: Sociológia, Vol. 45(5), 435-469.

Median (20I3). Prezidentská volba. Determinanty výsledku (Výzkum ve volebních dnech) [Die Präsidentenwahl. Determinanten des Wahlergebnisses (Erhebungen an den Wahltagen], Prag. Internet: http:// www.median.cz/docs/Median_PREZIDENT_II KOLO_determinanty_vysledku.pdf (Zugriff: 27.0I. 20I6).

Meyer, Thomas (2004). Die Identität Europas, Frankfurt am Main.

Müller, Karel B. (2012). Veřejná sféra, identita a dilema politizace: čtyřri syntetické teze $\mathrm{k}$ evropeizaci občanské společnosti [Öffentliche Sphäre, Identität und Dilemma der Politisierung: vier synthetische Thesen zur Europäisierung der Zivilgesellschaft], in: Politologická revue, Vol. I8(2), I2O-I36.

Novotný, Lukáš/Václav Houžvička (Hg.). 2007. Otisky historie $\mathrm{v}$ regionálních identitách obyvatel pohraničí, Sebedefinice a vzájemné vnímání Čechů a Němců v př́mém sousedství [Historische Prägestempel in regionalen Identitäten der Bewohner des Grenzlandes. Selbstdefinition und gegenseitige Wahrnehmung von Tschechen und Deutschen in direkter Nachbarschaft], Prag.

Novotný, Lukáš (2O12). Dekrety, odsun sudetských Němců v historické paměti Čechů. Výsledky reprezentativního dotazníkového šetření [Dekrete, Vertreibung der Sudetendeutschen im historischen Gedächtnis der Tschechen. Ergebnisse einer repräsentativen Befragung], in: Naše společnost, Vol. 8(2), 30-34.

Novotný, Lukáš/Matthias Niedobitek/Beate Neuss/Premysl Rosulek (Hg.) (2OI2). Kooperationsbeziehungen in der neuen Europäischen Union - unter besonderer Berücksichtigung des sächsisch-tschechischen Grenz- 
raums. Chemnitzer Schriften zur europäischen und internationalen Politik. Band 3, Hamburg.

Novotný, Lukáš (2013). Spolková republika Německo [Bundesrepublik Deutschland], Prag.

Novotný, Lukáš (2015). Von Konfrontation zum Konsens. Die Bilanz der deutsch-tschechischen Beziehungen seit 1989/90, in: Thieme, Tom (Hg.): 25 Jahre Systemwechsel in Osteuropa, Chemnitz, 175-192.

Olsen, Johan P. (2002). The Many Faces of Europeanization, in: Journal of Common Market Studies, Vol. 4O(5), $92 \mathrm{I}-952$.

Pavliček, Václav (Hg.) (2002). Benešovy dekrety [BenešDekrete], Prag.

Pešková, Jana (1998). Role vědomí v dějinách [Die Rolle des Bewusstseins in der Geschichte], Prag.

Perzi, Niklas (2003). Die Benes-Dekrete. Eine europäische Tragödie, St. Pölten/Wien.

Pomian, Krzysztof (1998). De l'histoire, partie de la mémoire, à la mémoire, objet d'histoire, in: Revue de Métaphysique et de Morale, Vol. I(I), 63-IIO.

Risse, Thomas (2010). A Community of Europeans? Transnational Identities and Public Spheres, Ithaca/ London.

Topalov, Christian/Amy Jacobs (2008). Maurice Halbwachs and Chicago Sociologists, in: Revue française de sociologie, Vol. 49(I), I87-2I4.

Vondra, Alexandr (20II). Jednání o deklaraci bylo složité [Die Verhandlungen über die Erklärung waren schwierig], in: Marek Loužek (Hg.): Česko-německá deklarace. Deset let poté. [Die Deutsch-Tschechische Erklärung. Zehn Jahre danach], Prag, II-I4.

\section{Autor}

Lukáš Novotný ist Politikwissenschaftler und Soziologe. Er promovierte an der TU Chemnitz zum Thema der aktuellen deutsch-tschechischen Vergangenheitsdiskurse. Heute lehrt er an der Jan Evangelista Purkyně Universität in Ústí nad Labem sowie an der Karls-Universität Prag und beschäftigt sich schwerpunktmäßig mit den politischen Systemen der deutschsprachigen Länder. 